\title{
Graphene-based Inks for Flexible Electronics: Effect of Surfactant and Various Types of Solvents
}

\author{
Mohd Saidina Dandan Satia, ${ }^{1,2}$ Mariatti Mustapha, ${ }^{1 *}$ Syazana Ahmad Zubir, ${ }^{1}$ \\ Sébastien Fontana ${ }^{2}$ and Claire Hérold ${ }^{2}$ \\ ${ }^{1}$ School of Materials and Mineral Resources Engineering, Universiti Sains Malaysia, \\ Engineering Campus, 14300 Nibong Tebal, Pulau Pinang, Malaysia \\ ${ }^{2}$ Institut Jean Lamour, Campus Artem, 2 allée Guinier, BP 50840, \\ 54011 Nancy Cedex, France \\ *Corresponding author: mariatti@usm.my
}

Published online: 25 December 2019

To cite this article: Dandan Satia, M. S. et al. (2019). Graphene-based inks for flexible electronics: Effect of surfactant and various types of solvents. J. Phys. Sci., 30(Supp. 2), 167-178, https://doi.org/10.21315/jps2019.30.s2.14

To link to this article: https://doi.org/10.21315/jps2019.30.s2.14

\begin{abstract}
The promising and extraordinary properties of graphene has attracted significant interest from many researchers especially in various electronic applications. The main aim of this work is to develop graphene-based ink with excellent stability and electrical properties for flexible electronics. Graphene foam $(G F)$ was synthesised by using solvothermal method. GF exhibited multi layers of graphene, good in-plane crystallite quality, a low carbon impurity and relatively low sodium carbonate content. GF was further dispersed into various types of solvents. Results showed that GF dispersed in dimethyl sulfoxide (DMF) and N-methylpyrrolidone (NMP) exhibited good stability. However, these solvents suffer from highly toxic which may damage fertility or the unborn child (hazard code = H360). Besides that, GF dispersed in distilled water with an addition of sodium dodecyl sulfate (SDS) as a surfactant presented good stability as compared to GF ink without surfactant and can be further used as the conductive ink for printable electronics.
\end{abstract}

Keywords: Graphene foam, solvothermal reaction method, surfactant, stability, conductive ink

\section{INTRODUCTION}

Graphene, a novel 2D carbon nanomaterial, has provoked much research interest recently due to its high electrical conductivity of up to $6000 \mathrm{~S} \mathrm{~cm}^{-1}$, superior mechanical properties with Young's Modulus of $1 \mathrm{TPa}$ and ultimate strength 
of $130 \mathrm{GPa} .{ }^{1-3}$ Previous researchers have reported that conductive ink made of graphene provide alternative to conventional carbon-based and metal inks that have shown low conductivity, poor processability and flexibility. Metals such as silver $(\mathrm{Ag})$, gold $(\mathrm{Au})$, copper $(\mathrm{Cu})$ and aluminium (Al) have shown excellent electrical properties. However, $\mathrm{Ag}$ and $\mathrm{Au}$ are expensive to be utilised in large quantities, while $\mathrm{Cu}$ and $\mathrm{Al}$ are easily oxidised. Due to that, high-yield production of graphene is required.

Several methods for the mass production of graphene such as chemical vapour deposition (CVD), liquid phase exfoliation (LPE), graphite oxide route such as graphene oxide (GO) and reduced graphene oxide (RGO), electrochemical route and solvothermal method have been widely studied. ${ }^{4-8} \mathrm{LPE}$ method is considered to be one of the simplest methods and yields larger quantities of graphene. However, this method introduces defects in the graphene layers which may not be suitable to be used as conductive inks. ${ }^{9-11}$ Choucair et al. in their study reported that the synthesis of graphene by solvothermal reaction between ethanol and sodium offers several advantages including high quality of few-layer graphene, high yield of graphene and the graphene-based powder is easy to be handled. ${ }^{12}$

Apart of this, there is still a problem in processing graphene, in particular graphene's poor colloidal stability in most common solvents. ${ }^{13,14}$ To date, there are limited studies that investigate the effect of different types of dispersion solvent on the stability of graphene ink. However, few good solvents including dimethyl sulfoxide (DMF) and N-methylpyrrolidone (NMP) have been widely discovered and can possibly be used to disperse graphene. Table 1 presents various types of solvents used for graphene ink dispersions. ${ }^{15-21}$

Table 1: Various types of solvents used to disperse graphene.

\begin{tabular}{llcc}
\hline $\begin{array}{l}\text { Conductive } \\
\text { material }\end{array}$ & Solvent(s) & $\begin{array}{c}\text { Electrical } \\
\text { conductivity }\end{array}$ & Ref. \\
\hline Graphene & NMP & $100-3000 \mathrm{~S} \mathrm{~m}^{-1}$ & 15,16 \\
Graphene & $\begin{array}{l}\text { NMP with ethyl cellulose (EC), cyclohexanone/ } \\
\text { terpineol, EC ethanol/terpineol, EC and } \\
\text { cyclohexanone }\end{array}$ & $3400-25000 \mathrm{~S} \mathrm{~m}^{-1}$ & $17-19$ \\
Graphene & $\begin{array}{l}\text { Water and sodium dodecylbenzene sulphonate } \\
\text { (SDBS) as surfactant }\end{array}$ & $1500 \mathrm{~S} \mathrm{~m}^{-1}$ & 20 \\
Graphene & Dimethyl-acetamide & $10^{5} \mathrm{~S} \mathrm{~m}^{-1}$ & 21 \\
\hline
\end{tabular}


In the present study, graphene foam (GF) as the graphene-like material was synthesised by using solvothermal reaction method and GF dispersed into various types of common solvents with and without surfactant were produced using sonication method. Properties of synthesised GF and graphene-based inks were investigated.

\section{EXPERIMENTAL}

\subsection{Synthesis of GF}

GF was prepared using solvothermal method according to the method reported by Speyer et al. ${ }^{11}$ In this method, ethanol and metallic sodium were mixed together (1:1 stoichiometric mixture) in a Parr autoclave under inert atmosphere. A pressure of 100 bar was introduced in the reactor by injection of nitrogen. The autoclave was heated at $220^{\circ} \mathrm{C}$ for $72 \mathrm{~h}$ (the internal pressure reaches $200 \mathrm{bar}$ ) and finally was cooled down to room temperature. The sodium ethoxide solvothermal product was collected under argon atmosphere.

Solvothermal product was placed in an Inconel crucible, inside a vertical tubular oven. The oven was heated at temperature of $850^{\circ} \mathrm{C}$ with a heating rate of $20^{\circ} \mathrm{C}$ $\min ^{-1}$ for $8 \mathrm{~h}$ and cooled down to room temperature, without stopping the nitrogen flow. The reaction yielded carbon, sodium carbonate, sodium hydroxide and gaseous hydrocarbons.

The powder obtained after pyrolysis was sonicated for $15 \mathrm{~min}$ in ethanol, and washed with hydrochloric acid and distilled water in order to remove sodium carbonate and sodium hydroxide. The washed sample was finally dried at $100^{\circ} \mathrm{C}$ for $24 \mathrm{~h}$. The final yield was varied from $2 \mathrm{wt} \%$ to $6 \mathrm{wt} \%$ (approximately $250 \mathrm{mg}$ ).

\subsection{Production of Graphene-based Ink}

For graphene ink, $50 \mathrm{mg}$ of graphene powder and $50 \mathrm{ml}$ of solvent were poured onto a $100 \mathrm{ml}$ beaker. Various types of solvents were used, including NMP, DMF, distilled water and surfactant such as sodium dodecyl sulfate (SDS). The mixture was sonicated for $1 \mathrm{~h}$ with $50 \%$ amplitude and 0.5 sonication cycle at room temperature. The solution was centrifuged at $4000 \mathrm{rpm}$ for $10 \mathrm{~min}$. The supernatant was collected. Various types of inks are listed in Table 2. 
Table 2: Description of the sample codes for various types of inks.

\begin{tabular}{ll}
\hline Ink & Sample code \\
\hline GF dispersed in distilled water & G ink (no surfactant) \\
GF dispersed in distilled water with SDS as surfactant & G ink (with surfactant) \\
GF dispersed in DMF solvent & G ink (DMF) \\
GF dispersed in NMP solvent & G ink (NMP) \\
\hline
\end{tabular}

\subsection{Characterisation Techniques}

X-ray powder diffraction (XRD) measurement was performed using Bruker D8 Advance Diffractometer with molybdenum (Mo) $\mathrm{K} \alpha$ radiation $(\lambda=0.7093)$ in a wide range of $2 \theta(10 \leq 2 \theta \leq 30)$. The crystallite size, $L_{c}$ was calculated using the Debye-Scherrer equation (Equation 1). $\theta$ represents the Bragg angle and $\beta$ represents the full-width at half maximum (FWHM) of the (002) peak. The morphology of GF was characterised by using high resolution transmission electron microscope (HRTEM) (Jeol ARM 200F). Raman spectroscopy was carried out by using Horiba Jobin-Yvon LabRAM HR800 spectrometer operating at the red radiation $(\lambda=633 \mathrm{~nm})$ on the spectral ranges $850-1800 \mathrm{~cm}^{-1}$ (D and $\mathrm{G}$ bands) and 2500-2850 $\mathrm{cm}^{-1}$ (2D band). Thermogravimetric analysis (TGA) was done using the Setsys Evolution Setaram thermobalance. The sample was heated up to $1000^{\circ} \mathrm{C}$ with the heating rate of $3^{\circ} \mathrm{C} \mathrm{min}^{-1}$ and conducted under dry air. The surface potential and particle size measurement of the inks were examined using Zetasizer Malvern. Observation test was carried out via visual inspection.

$$
\text { Crystallite size, } L_{c}=\frac{0.91 \lambda_{M O}}{\beta \cos (\theta)}
$$

\section{RESULTS AND DISCUSSION}

\subsection{Properties of Graphene Foam}

Figure 1 shows HRTEM micrographs of the synthesised GF. From the micrographs, it can be observed that GF sample exhibited semi-crystalline structure as amorphous carbon can be seen by observing of highly buckled GF particle. The interlayer spacing was measured at higher magnification with a value of $0.34 \mathrm{~nm}$ which in agreement with a graphitic stacking and consists of multi-layer graphene. It should be noted that HRTEM provides information only on a small part of the sample which was not sufficient to explain the trend and thickness of the sample. 


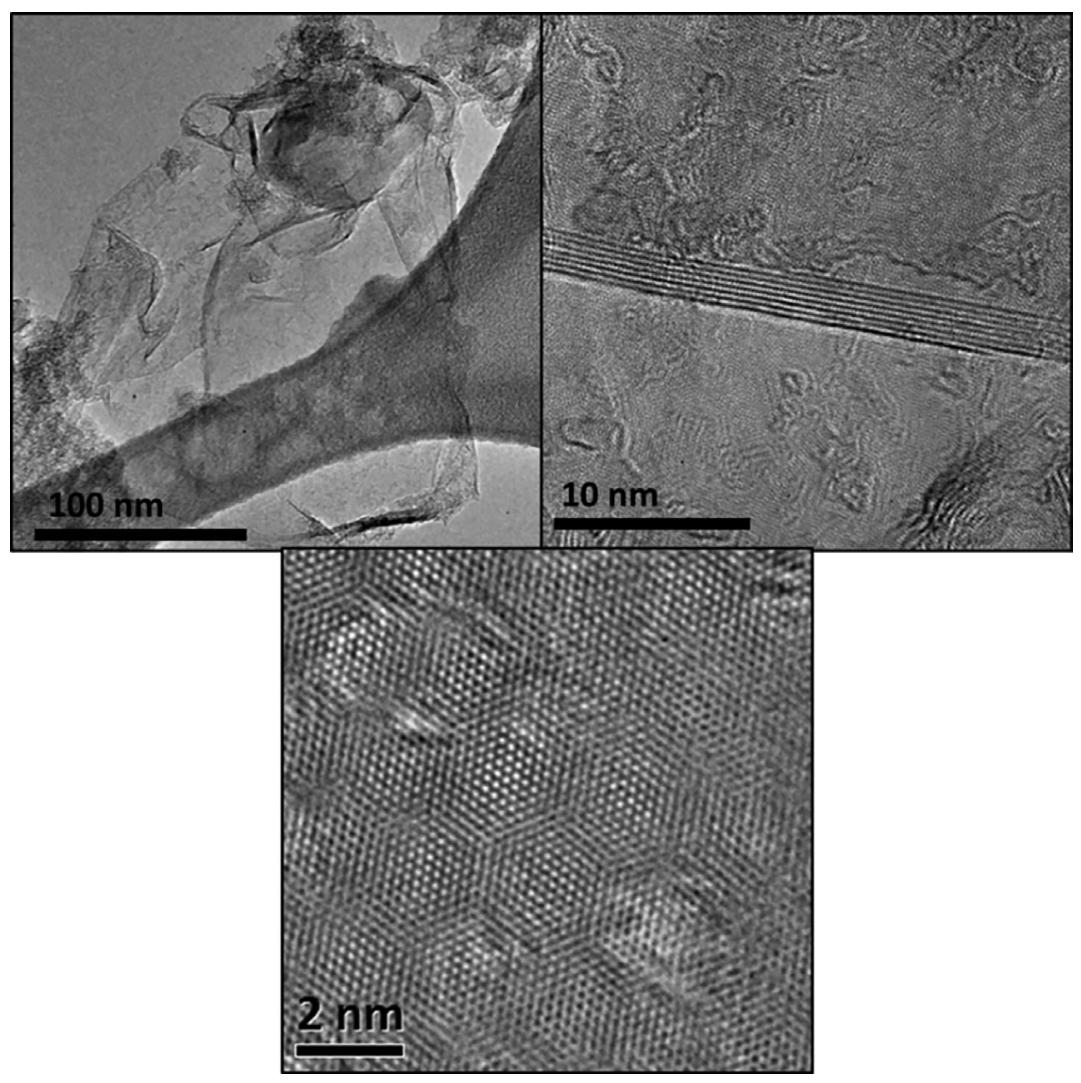

Figure 1: HRTEM micrographs of the synthesised GF at different magnifications.

GF synthesised using solvothermal reaction method has been characterised by XRD measurement, as shown in Figure 2. It shows that the sample presents supplementary crystalline phases: sodium carbonate (space group $C 12 / \mathrm{m} 1$, $\mathrm{a}=891 \mathrm{pm}, \mathrm{b}=524 \mathrm{pm}, \mathrm{c}=605 \mathrm{pm},=101.32$ ) resulting from the reaction of pyrolysis and orthorhombic graphite (space group Cmma, $\mathrm{a}=458 \mathrm{pm}, \mathrm{b}=530 \mathrm{pm}$, $\mathrm{c}=563 \mathrm{pm}$ ). Note that sodium carbonate was still present and trapped in the sample due to the washing step with concentrated hydrochloric acid. At (002) Bragg peak, the value of the coherence length along the $\mathrm{c}$ axis of graphite and an augmentation of the number of stacked layers can be calculated by using the Debye-Scherrer equation. The Debye-Scherrer equation gives median value, which is overestimated in comparison with the very thin regions observed by HRTEM. The calculated $L_{c}$ value is $3.8 \mathrm{~nm}$. 


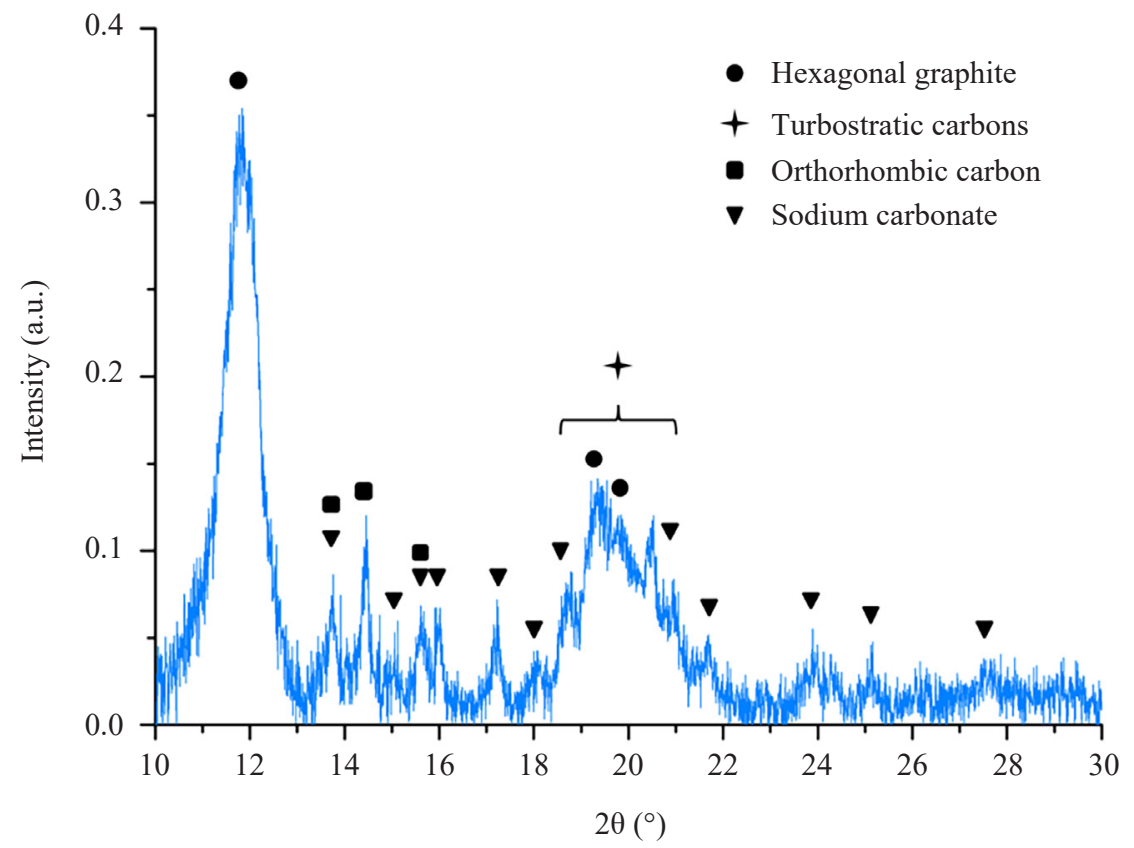

Figure 2: XRD pattern of the synthesised GF.

Raman spectroscopy is a versatile tool to get useful information about carbonaceous materials. There are three disorder-related bands that are prominent for Raman spectra of graphene, including the D peak at around $1350 \mathrm{~cm}^{-1}, \mathrm{G}$ peak at around $1580 \mathrm{~cm}^{-1}$ and 2D peak at around $2700 \mathrm{~cm}^{-1} \cdot{ }^{22,23}$ By using the Raman spectra, it is also possible to identify the amounts of defects and their types (D band), in-plane $\mathrm{sp}^{2}$ hybridised carbon atoms ( $\mathrm{G}$ band), stacking order and the number of layers (2D band) in graphene samples. ${ }^{24}$ Figure 3 shows the Raman spectrum of synthesised GF. The sample shows 3 distinct peaks at approximately $1329 \mathrm{~cm}^{-1}$, $1589 \mathrm{~cm}^{-1}$ and $2663 \mathrm{~cm}^{-1}$. The number of layers could be derived from the ratio of peak intensities for $2 \mathrm{D}$ and $\mathrm{G}\left(I_{2 \mathrm{D}} / I_{\mathrm{G}}\right)$. The ratio of $I_{2 \mathrm{D}} / I_{\mathrm{G}}$ for GF was 0.42 , indicating that GF consists of multi-layer graphene. Therefore, the Raman spectrum of synthesised GF shown in Figure 3 confirms the formation of graphene.

TGA under dry air at heating rate of $3^{\circ} \mathrm{C} \mathrm{min}^{-1}$ was performed to examine purity, the onset degradation temperature, $\mathrm{T}_{\text {onset }}$ and final mass loss at $1000^{\circ} \mathrm{C}$. The TG and dTG curves are shown in Figure 4. From the TG curve, it can be explained that synthesised graphene sample exhibits a major loss in between $300^{\circ} \mathrm{C}$ to $450^{\circ} \mathrm{C}$ and only one mass loss was observed which indicates that the sample is free from impurities. However, the final mass loss at $1000^{\circ} \mathrm{C}$ was not equal to $100 \%$ due to the presence of residual sodium carbonate which degrades above $750^{\circ} \mathrm{C}$ to 
form sodium oxide, $\mathrm{Na}_{2} \mathrm{O}$. This result is parallel with the XRD results in which sodium carbonate was detected in the sample. From the dTG curve, the synthesised graphene has a $\mathrm{T}_{\text {onset }}$ value of $410^{\circ} \mathrm{C}$.

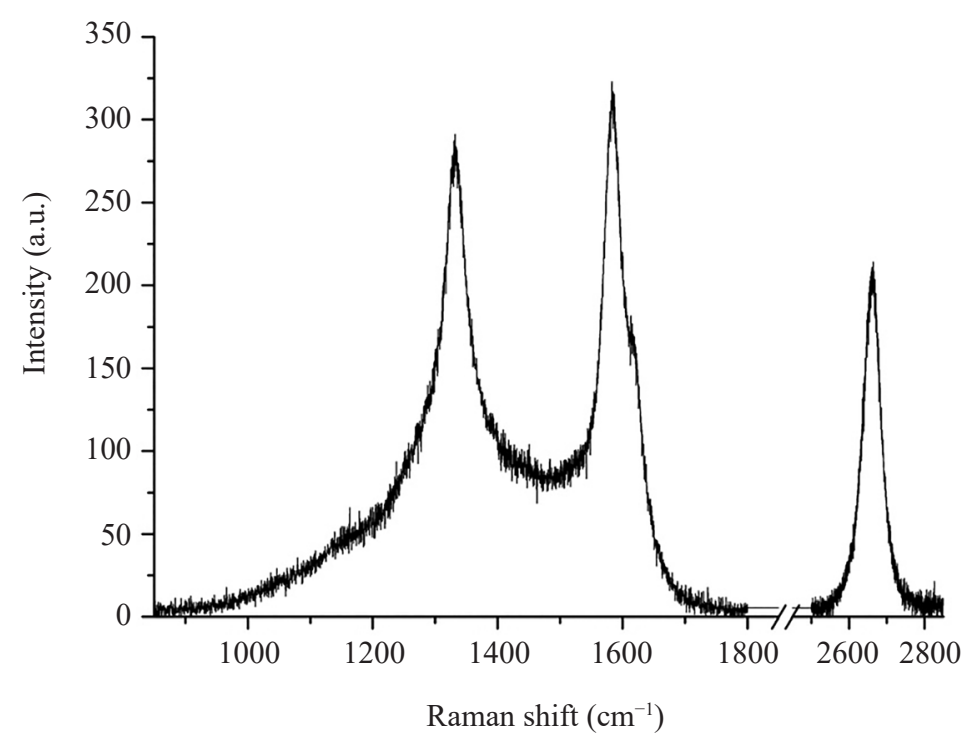

Figure 3: Raman spectrum of the synthesised GF.

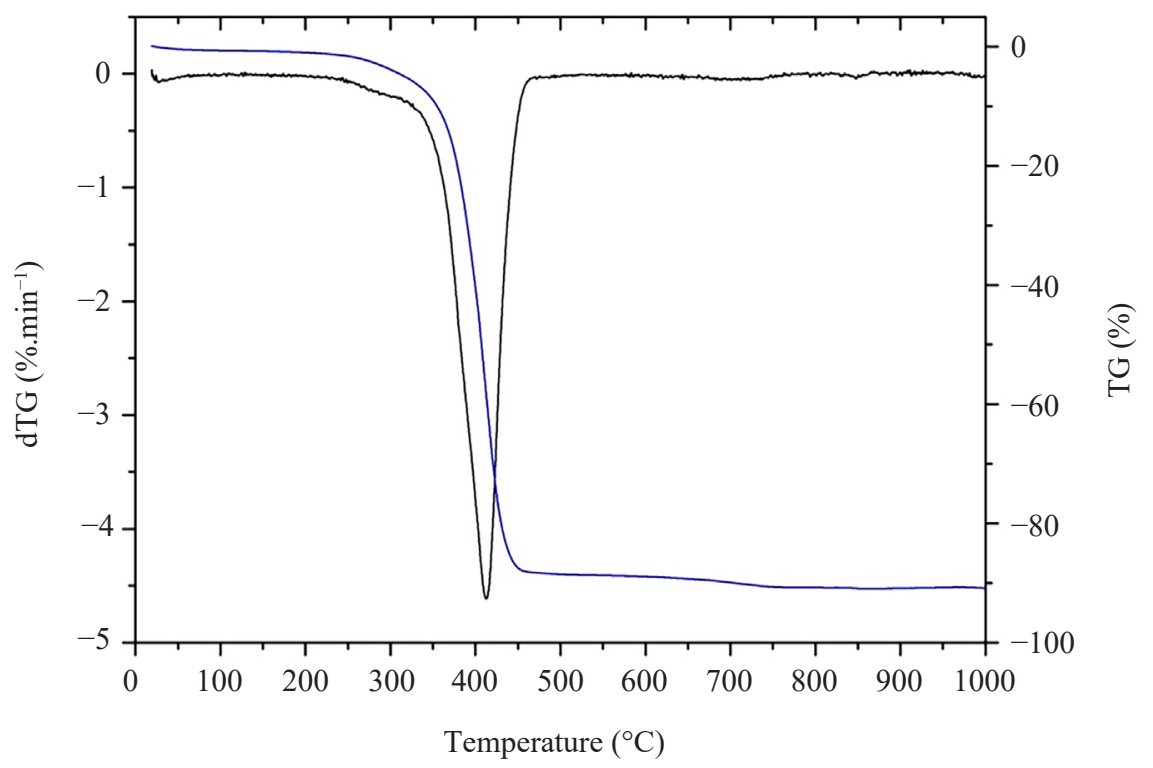

Figure 4: TG and dTG curves of the synthesised GF. 


\subsection{Stability of Graphene-based Inks}

Figure 5 shows the surface potential value of GF dispersed in distilled water with and without SDS as surfactant at different $\mathrm{pH}$ values. According to $\mathrm{Li}$ et al., zeta, $\zeta$-potential can be described as the indicator of stability of a colloidal system and the theoretical limit is $|30 \mathrm{mV}| .^{25}$ The colloidal solution is considered to be stable at $\zeta$-potential value higher than $30 \mathrm{mV}$ or lower than $-30 \mathrm{mV}$. From Figure 5, it can be observed that sample $G$ ink (no surfactant) has the isoelectric (IEP) point at $\mathrm{pH}$ 9. Value of IEP was obtained by measuring the $\zeta$-potential as a function of $\mathrm{pH}$ and identifying the $\mathrm{pH}$ at which the $\zeta$-potential value crosses zero. IEP often refers at which the particle surface charge is zero and the system is not particularly stable. Besides that, sample $\mathrm{G}$ ink (no surfactant) was not stable at all $\mathrm{pH}$ as the $\zeta$-potential values were in between $-12 \mathrm{mV}$ to $23 \mathrm{mV}$. Therefore, surfactant or surface treatment was suggested to improve the dispersion stability of $\mathrm{G}$ ink.

As can be seen from the figure, sample $\mathrm{G}$ ink (with surfactant) was stable at all $\mathrm{pH}$, no isoelectric (IEP) point and the values of $\zeta$-potential were in between -69 to -45 $\mathrm{mV}$. It can be seen that the addition of SDS as a surfactant improved the dispersion stability of G ink. For comparison purpose, G ink (NMP) and G ink (DMF) showed $\zeta$-potential values of -33 and $-17 \mathrm{mV}$, respectively. The $\zeta$-potential was measured at the current $\mathrm{pH}$ of $\mathrm{G}$ ink (NMP) and $\mathrm{G}$ ink (DMF) with the measured $\mathrm{pH}$ values of 6 and 7, respectively. This indicated that G ink (NMP) was stable.

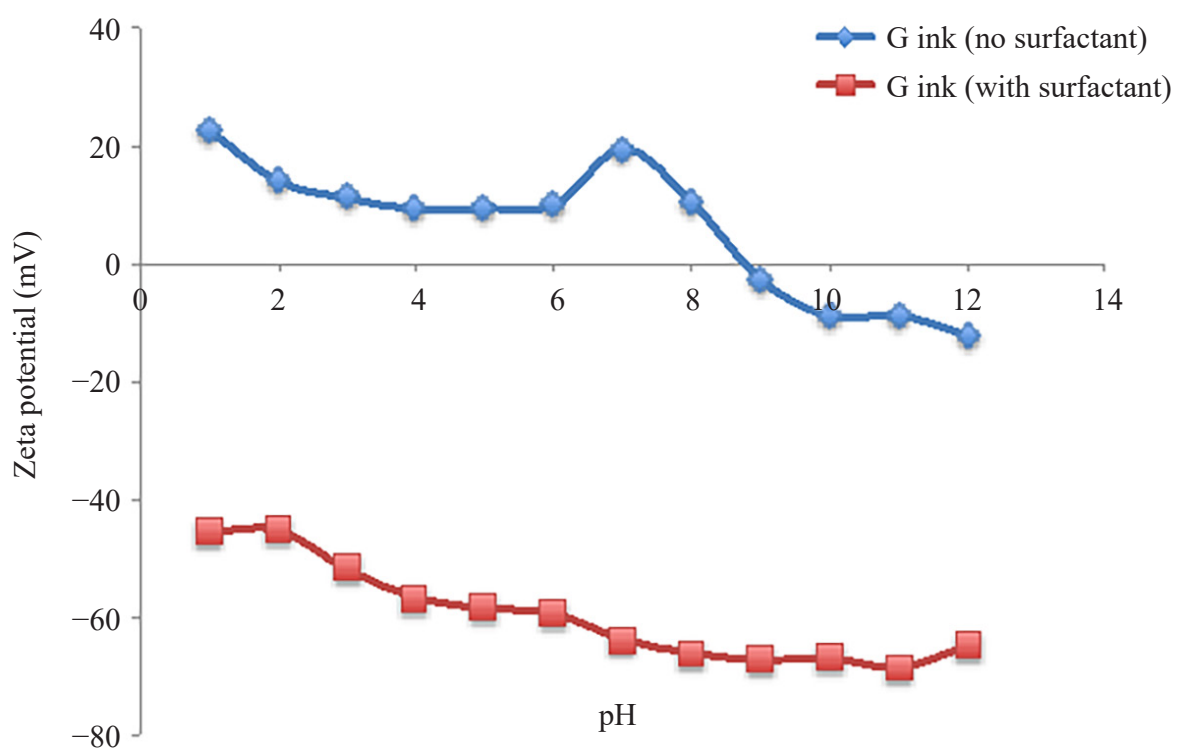

Figure 5: Surface potential of graphene ink with and without SDS as surfactant. 
Observation test was carried out in order to further investigate the dispersion stability of the $G$ inks. Figure 6 shows the photographs of graphene ink for: (a) no surfactant; (b) with surfactant; (c) DMF; and (d) NMP monitored at different periods of time, between day 1 (after sonication), day 3 and day 7 . Illustration in Figure 6(i) shows that sample G ink (no surfactant) was not stable and completely settled at the bottom in less than a week as shown in Figure 6 (ii and iii) due to the strong van der Waals attraction and has been proven by sedimentation of GF near the bottom of the bottle. Meanwhile, the other samples such as G ink (with surfactant), G ink (DMF) and G ink (NMP) were stable up to a week as also shown in Figure 6. The dispersion stability of prepared inks has been monitored at greater than 2 weeks and no precipitates have been observed yet for G ink (with surfactant), G ink (NMP) and G ink (DMF) samples. However, the toxic effects of NMP and DMF in humans need to be taken into consideration. According to Byrne et al., DMF and NMP may damage fertility or the unborn child (hazard code $=\mathrm{H} 360){ }^{26}$

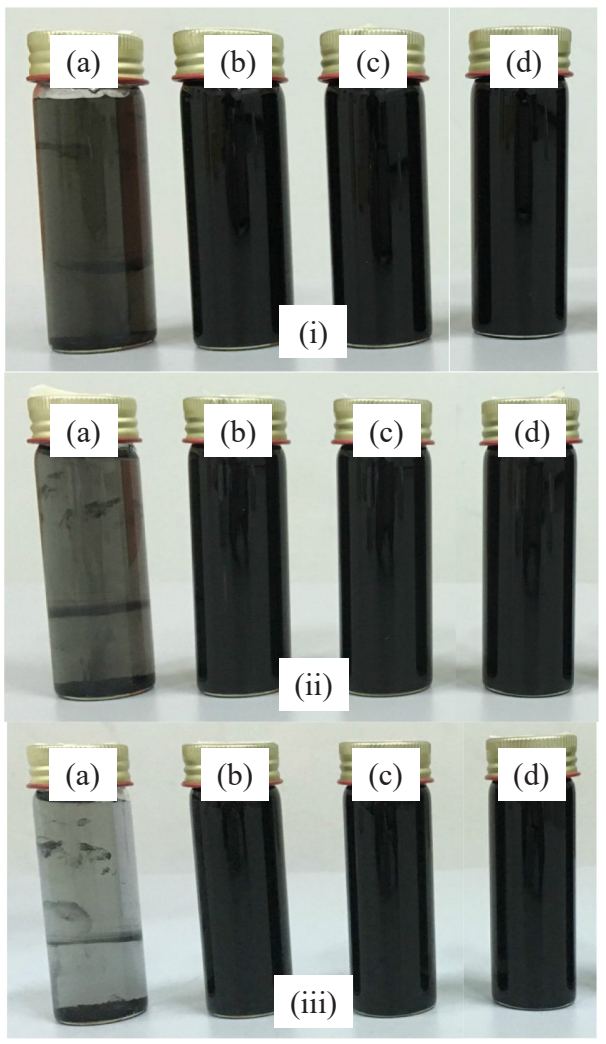

Figure 6: Images of graphene ink for (a) no surfactant, (b) with surfactant, (c) DMF, and (d) NMP. Images were taken on (i) day 1, (ii) day 3, and (iii) day 7. 
Figure 7 presents the average particle sizes of $\mathrm{G}$ inks over a long period of time. It was observed that the average particle sizes for samples G ink (DMF) did not increase throughout the analysing process and the average values were relatively stable than those of G ink (NMP) and G ink (with surfactant).

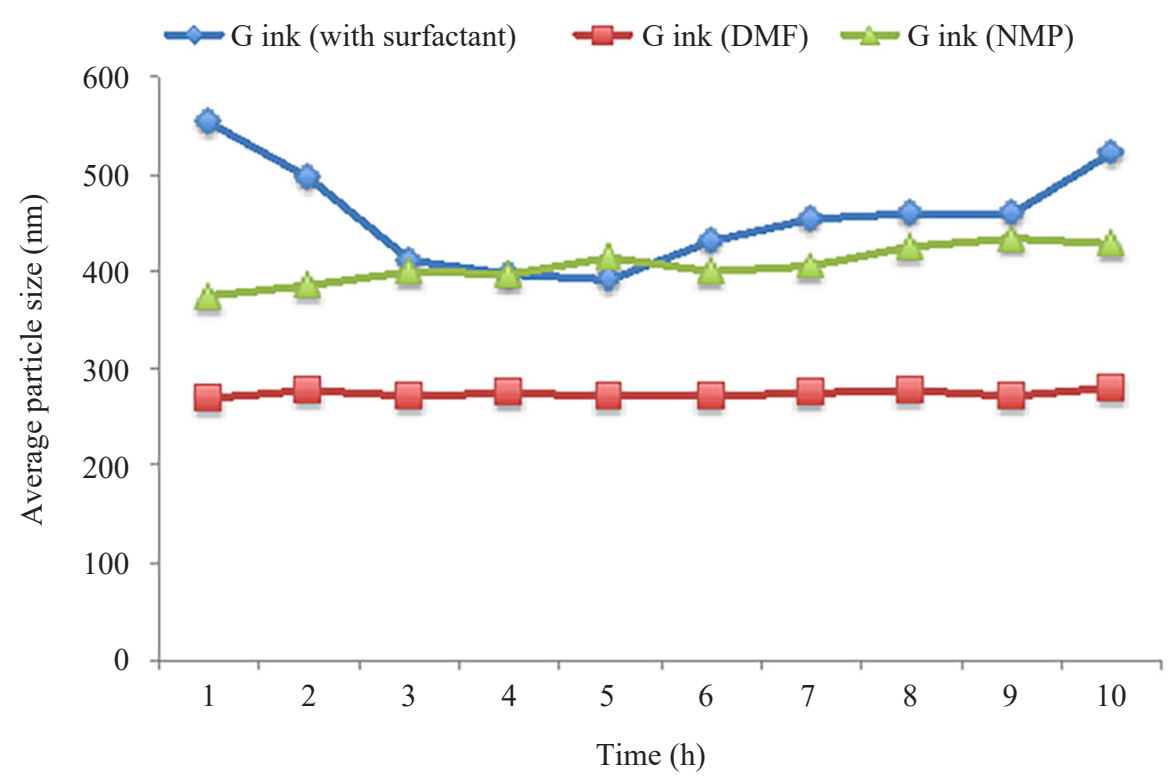

Figure 7: Average particle sizes of graphene inks over a long period of time.

\section{CONCLUSION}

We have synthesised GF using solvothermal reaction method and dispersed GF into various types of solvents. It was found that $G$ ink dispersed in NMP, DMP and distilled water (with surfactant) exhibited good stability up to several weeks. However, NMP and DMF solvents suffer from toxicity. G ink (with surfactant) has been chosen to be used as conductive ink for printing electronics applications.

\section{ACKNOWLEDGEMENTS}

The authors would like to acknowledge the support from Universiti Sains Malaysia, Université de Lorraine and the Ministry of Education Malaysia through Fundamental Research Grant Scheme Malaysia's Rising Star Awards (FRGS MRSA) for granting the research fund for this project (project no. 6071385). 


\section{REFERENCES}

1. Singh, V. et al. (2011). Graphene based materials: Past, present and future. Progr. Mater. Sci., 56, 1178-1271, https://doi.org/10.1016/j.pmatsci.2011.03.003.

2. Chia, J. S. Y. et al. (2014). A novel one step synthesis of graphene via sonochemicalassisted solvent exfoliation approach for electrochemical sensing application. Chem. Eng. J., 249, 270-278, https://doi.org/10.1016/j.cej.2014.03.081.

3. Novoselov, K. S. et al. (2004). Electric field effect in atomically thin carbon films. Sci., 306, 666-669, https://doi.org/10.1126/science.1102896.

4. Dhand, V. et al. (2013). A comprehensive review of graphene nanocomposites: Research status and trends. J. Nanomater., 14, Article ID 763953, https://doi. org/10.1155/2013/763953.

5. Saidina, D. S. et al. (2019). Recent development of graphene-based ink and other conductive material-based inks for flexible electronics. J. Electron. Mater., 48(6), 3428- 3450, https://doi.org/10.1007/s11664-019-07183-w.

6. Aisyah, A. N. et al. (2018). Effect of frequency and number of piezoelectric probes in sonication- assisted exfoliation of graphite layers into graphene oxide. J. Phys. Sci., 29, 121-135, https://doi.org/10.21315/jps2018.29.2.8.

7. Kong, H. X. (2013). Hybrids of carbon nanotubes and graphene/graphene oxide. Curr. Opin. Solid State Mater. Sci., 17, 31-37, https://doi.org/10.1016/j. cossms.2012.12.002.

8. Tkalya, E. E. et al. (2012). The use of surfactants for dispersing carbon nanotubes and graphene to make conductive nanocomposites. Curr. Opin. Coll. Interf. Sci., 17, 225-232, https://doi.org/10.1016/j.cocis.2012.03.001.

9. Stankovich, S. et al. (2007). Synthesis of graphene-based nanosheets via chemical reduction of exfoliated graphite oxide. Carbon, 45, 1558-1565, https://doi. org/10.1016/j.carbon.2007.02.034.

10. Bagri, A. et al. (2010). Structural evolution during the reduction of chemically derived graphene oxide. Nat. Chem., 2, 581-587, https://doi.org/10.1038/ nchem.686.

11. Speyer, L. et al. (2015). Multi-scale characterization of graphenic materials synthesized by a solvothermal-based process: Influence of the thermal treatment. Solid State Sci., 50, 42-51, https://doi.org/10.1016/j.solidstatesciences.2015.10. 009.

12. Choucair, M., Thodarson, P., \& Stride, J. A. (2009). Gram-scale production of graphene based on solvothermal synthesis and sonication. Nat. Nanotech., 4, 30-33, https://doi.org/10.1038/nnano.2008.365.

13. Ayán-Varela, M. et al. (2014). A quantitative analysis of the dispersion behavior of reduced graphene oxide in solvents. Carbon, 75, 390-400, https://doi.org/10.1016/j. carbon.2014.04.018.

14. Johnson, D. W., Dobson, B. P. \& Coleman, K. S. (2015). A manufacturing perspective on graphene dispersions. Curr. Opin. Coll. Interf. Sci., 20, 367-382, https://doi.org/10.1016/j.cocis.2015.11.004. 
15. Torrisi, F. et al. (2012). Inkjet-printed graphene electronics. ACS Nano, 6, 2992-3006, https://doi.org/10.1021/nn2044609.

16. Finn, D. et al. (2013). Inkjet deposition of liquid-exfoliated graphene and $\mathrm{MoS}_{2}$ nanosheets for printed device applications. J. Mater. Chem. C, 2, 925-932, https://doi.org/10.1039/c3tc31993h.

17. Secor, E. B. et al. (2013). Inkjet printing of high conductivity, flexible graphene patterns. J. Phys. Chem. Lett., 4(8), 1347-1351, https://doi.org/10.1021/jz400644c.

18. Li, J. et al. (2013). Efficient inkjet printing of graphene. Adv. Mater., 25, 39853992, https://doi.org/10.1002/adma.201300361.

19. Gao, Y. et al. (2014). Inkjet printing patterns of highly conductive pristine graphene on flexible substrates. Ind. Eng. Chem. Res., 53, 16777-16784, https://doi.org/10.1021/ie502675z.

20. Lotya, M. et al. (2009). Liquid phase production of graphene by exfoliation of graphite in surfactant/water solutions. J. Am. Chem. Soc., 131(10), 3611-3620, https://doi.org/10.1021/ja807449u.

21. Blake, P. et al. (2008). Graphene-based liquid crystal device. Nano Lett., 8, 1704-1708, https://doi.org/10.1021/n1080649i.

22. Klar, P. et al. (2013). Raman scattering efficiency of graphene. Phys. Rev. B, 87, 205435, https://doi.org/10.1103/PhysRevB.87.205435.

23. Ferrari, A. C. et al. (2006). Raman spectrum of graphene and graphene layers. Phys. Rev. Lett., 9, 187401-187404, https://doi.org/10.1103/PhysRevLett.97. 187401 .

24. Cançado, L. G. et al. (2008). Measuring the degree of stacking order in graphite by Raman spectroscopy. Carbon, 46, 272-275, https://doi.org/10.1016/j. carbon.2007.11.015.

25. Li, D. et al. (2008). Processable aqueous dispersions of graphene nanosheets. Nat. Nanotechnol., 3, 101-105, https://doi.org/10.1038/nnano.2007.451.

26. Byrne, F. P. et al. (2016). Tools and techniques for solvent selection: Green solvent selection guides. Sustain. Chem. Process, 4, 7, https://doi.org/10.1186/s40508016-0051-z. 